\title{
Game Theory-Based Minimization of the Ostracism Risk in Construction Companies
}

\author{
Dimosthenis Kifokeris $^{1}$ (D) and Yiannis Xenidis ${ }^{2, *}$ (D) \\ 1 Department of Architecture and Civil Engineering, Chalmers University of Technology, \\ 41296 Gothenburg, Sweden; dimkif@chalmers.se \\ 2 Department of Civil Engineering, Aristotle University of Thessaloniki, 54124 Thessaloniki, Greece \\ * Correspondence: ioxen@civil.auth.gr; Tel.: +30-2310-995525
}

check for updates

Citation: Kifokeris, D.; Xenidis, Y. Game Theory-Based Minimization of the Ostracism Risk in Construction Companies. Sustainability 2021, 13, 6545. https://doi.org/10.3390/ su13126545

Academic Editor:

Jurgita Antuchevičienè

Received: 18 April 2021

Accepted: 4 June 2021

Published: 8 June 2021

Publisher's Note: MDPI stays neutral with regard to jurisdictional claims in published maps and institutional affiliations.

Copyright: (c) 2021 by the authors. Licensee MDPI, Basel, Switzerland. This article is an open access article distributed under the terms and conditions of the Creative Commons Attribution (CC BY) license (https:/ / creativecommons.org/licenses/by/ $4.0 /)$.

\begin{abstract}
Strategic and managemerial decision-making in an organization can have a crucial effect for the whole entity; however, it rarely involves the organization's employees evenly at the different organizational levels. The result is—what is addressed in this paper as—the ostracism risk, namely the risk accruing from the lack of satisfaction of underprivileged employees' groups during the decision-making process. The ostracism risk could jeopardize the organization's integrity and therefore requires effective treatment. This paper aims at verifying a conceptual approach, which is proposed as a methodology for assessing the probability of organizational cooperation when deciding under risk, thus minimizing ostracism risk. The proposed approach is based on organizational and human resources management (HRM) theories and is contextualized for construction through the understanding of systems theory. The proposed methodology presents a potential modelling via game theory of a medium-sized construction company that is organized according to Mintzberg's organizational model. The utilization of the bounded Pareto distribution is presented as an approach of the model's probabilistic processing, and the potential for estimating the probabilities to adopt a favorable cooperational decision is verified. The paper concludes with the reference to the next steps required for the methodology's validation and further improvement.
\end{abstract}

Keywords: risk analysis; game theory; decision-making; organization theory; Pareto principle

\section{Introduction}

Positive or negative outcomes of decisions regarding organizational structure, function, and coordination (e.g., corporate mergers), are affiliated with the so-called external risk, which accrues from the decisions themselves and is distributed among the personnel organizational components, influencing them accordingly [1]. However, "ostracism risk" can be equally or even more critical than the external; "ostracism risk" is defined in this paper as the risk accruing from the lack of satisfaction of possibly underprivileged constituent personnel components (individuals or groups) during the process of the decision-making. The ostracism risk, along with the outcomes of the external risk, can manifest into situations detrimental to the organizational integrity in the short-, medium- and long-term (e.g., complaints, worsening job performance) [2]. On the contrary, even if the external risk is critically maximized, effective cooperation among the personnel components of the decision-making team could minimize the ostracism risk, since the responsibility for the taken course of action and any positive or negative outcomes is more smoothly and evenly diffused [3].

To the best of the authors' knowledge, the impacts of the occurrence of ostracism risk have yet to be identified and assessed; in fact, the term as such is introduced for the first time in the current study. Nevertheless, there have been efforts discussing other concepts related to the same phenomenon. For example, Wach et al. based their research on the theoretical concept of strategic consensus, which according to them, describes a group decision-making process and participants' belief that the outcome (i.e., decision) is right [4]. 
The main benefits of strategic consensus are listed as increased organizational performance, cohesiveness, shared mentality, and trust [4]. Although the negative outcome related to the lack of strategic consensus can be easily identified (e.g., inefficient use of resources, disengagement, high employee turnover), they concluded that there are mixed empirical findings on the actual economic impact of the lack of strategic consensus [4].

Adler et al. described the organizational environment as "a networked structure of agents exchanging knowledge to ensure efficiency by using internal resources to make decisions" [5]. They acknowledged decision-making as a collective task and they proposed a modelling of the agents participating in this task and an estimation of their total competence for decision-making [5]. Although they referred to some successful examples (yet none of them in the construction industry context) of decentralized decision-making supported by autonomously organized teams of employees, they also did not present any figures about economic impacts [5].

Lin and Wu stated that research on collaborative relationships in the construction industry is dominated by network-based approaches that focus on measuring and estimating the networks' critical parameters (e.g., density) [6]. However, this technical approach leaves the non-measurable parameters of collaborative relationships in the process of decision-making (e.g., interests of the collaborative parties) out of the context.

Simu and Lidelöw presented empirical research on the perceptions of middle managers in construction companies on the relationship between operations strategies (e.g., resource-efficient, flow-efficient) and various fields of decision-making, such as standardization, production planning, supply chain, performance measuring, etc. [7]. Although they indirectly refer to several aspects that are affected by the organizational framework wherein decision-making takes place, their analysis lacks any reference, even indirect, to any kind of quantification of these effects.

The reviewed literature evidently indicates both the research interest on the organizational framework's relation and impact to decision-making as well as the diverse directions and perspectives followed in this course. Moreover, it reveals that despite the various aspects affected positively or negatively depending on the existence or lack of certain features and qualities, a clear quantification of these effects is largely lacking.

In this course of research, this paper investigates methodologically the probability of organizational cooperation during decision-making under risk, considering the maximization of individual, group and collective benefits, and the minimization of the ostracism risk. An exemplar organization is modelled via game theory, used for the definition of the distinct benefits and the illustration of the interactions among the decision-makers. The organizational aspects of the model emanate from organizational and human resources management (HRM) theories. The results taken from the said modelling are probabilistically processed using the bounded Pareto distribution. Thus, the methodology concludes by estimating the probability of the adoption of a favorable cooperational solution.

First, the theoretical background of the proposed methodology is displayed. Secondly, the research approach is summarily delineated. Then, the modelling of the exemplar reference organization (in the form of an "ideal type" construction company) takes place. Following is the probabilistic processing of the model and the derivation of the final results. Finally, the conclusions concerning the proposed methodology are drawn, and propositions for its further generalization, expansion, and improvement are mentioned.

\section{Methodological Context}

\subsection{Organizational and Human Resources Management Theories}

In the field of management, an organization is defined as a system of individuals or parts cooperating and coordinated to promote the common completion of purposes, achievement of targets, and production of results [8]. It is mainly characterized by its said purposes; the coordination efforts among its parts; its specific activity systems; the corresponding responsibilities, obligations, and hierarchies; its dependence on the human factor within; and its interaction with the external environment [8]. These characteristics 
are important in organizational decision-making and action-taking, especially when the decisions are largely under risk and alternative courses of action are considered, a most frequent case especially for construction companies, on which this paper focuses.

Such characteristics are dynamic and can be partly or wholly changed throughout the organizational operational existence, which is dynamic itself, fully influential to and influenced by its internal and external conditions [9], particularly for construction companies where business activities are largely human-dependent and extremely sensitive to variations of the socioeconomic environment.

Several methodologies have been proposed to quantify, customize, measure, statistically analyze, and standardize these characteristics. However, the human factor remains largely difficult to process, for a variety of reasons, spanning from psychological to characterological, and for both high- and low-ranking employees [10]. HRM dictates that the members of an organization should: (a) be active and substantial parts of it, considering their individual benefits as extensions of the collective one $[10,11]$, (b) be assisted in their actions by the systematic receipt, storage, use, and retrieval of information [12], (c) organize themselves in targeted groups discretizing, studying and resolving certain parts of a more extended project [13], and (d) hold definite organizational roles (not to be disturbed, otherwise the organization itself is disturbed) with a certain degree of jurisdiction and specialization [14]. HR structures in mature construction companies typically present all the above characteristics, with the possible exception of the effective information system, which is an asset that is still inconsistently found or even largely missing, especially for small and medium companies (SMEs).

The human factor blends with certain aspects of the current organizational theories that have formulated, among others, ways of discretization related to the organizational size (large, medium, or small, depending on the corresponding market environment and size of other organizations), legal form, and complexity [8]. However, the greatest part of the respective literature emphasizes the criteria regarding the character of the organizational objectives, and its defined paramount human resources components.

According to [8,15], and considering the relevant organizational objectives, ranking of the corresponding management issues, and key beneficiaries, construction companies fall in the category of enterprises, which aim at socially acceptable profitability. In this case, the key beneficiaries are the owners, while the main managerial issue is profit. Socially acceptable profitability is supposed to not only be earned legally and transparently, but also not be offensive to the common sense of justice, ethics, and egalitarianism.

Concerning the paramount human resources component, the Mintzberg framework [16-18], which is depicted in Figure 1, proposes that every organization, including construction companies, consists of some or all of the five following groups, which contain individuals with common organizational tasks and similar roles: the operating core (OC) (mainly tasked with the procreation of the respective services and/or products); the strategic apex (SA) (the highest level administrators, wholly responsible for the smooth organizational operation); the intermediate level (IL) or middle line (the intermediate administrators, actively linking the operating core to the strategic apex); the technostructure (TS) (the analysts developing and implementing standardization procedures in the organizational operations); and the support staff (SF) (the executives performing indirect tasks). 


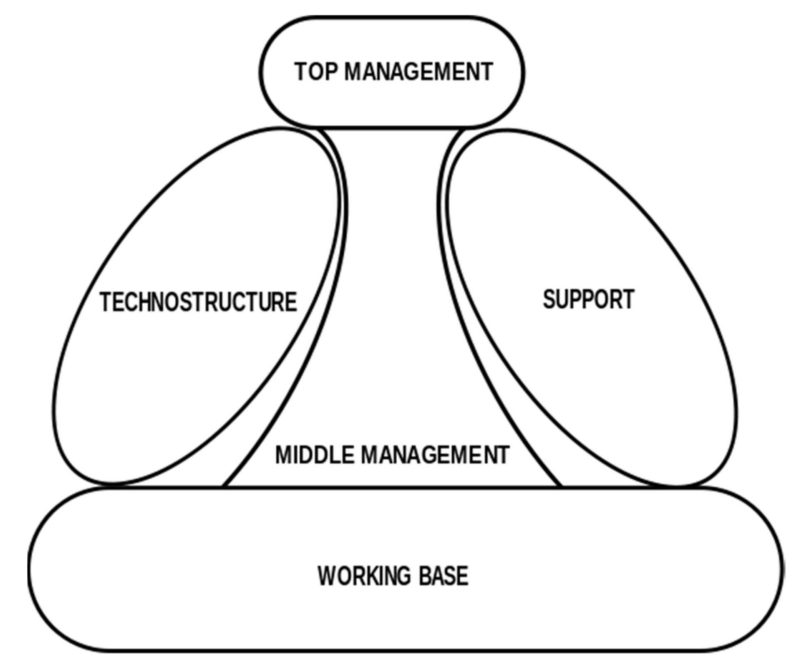

Figure 1. Mintzberg's organizational model reprinted with permission from ref. [19]. Copyright Year: 19 July 2011, Copyright Owner's Name: Jean-Christophe Benoist.

Considering the prevalence of a group over the rest, organizations can be discretized as [16-18]:

- Simply structured, with SA as the paramount group. They are usually small, without standardized operational procedures, and strongly influenced by a single person or a small group of general administrators (e.g., the founders of the organization).

Machine-bureaucratic, with TS as the paramount group. Appointed tasks in such organizations are extremely specialized. The standardization of the organizational procedures is extensive, and any workplace changes happen in a long-term plan. All decisions are made by an administrative group.

- Professional-bureaucratic, with OC as the paramount group. The standardization of the organizational procedures is extensive as well, albeit the employees are highly specialized themselves and capable of demarcated self-acting.

- Divisionalized, with IL as the paramount group. They consist of distinctive groups implementing certain production lines in a semiautonomous way, while the respective administration is exclusively focused in large-magnitude decision-making.

- Ad hoc, with SF as the paramount group. They are not structurally standardized, and function as organic models including team building among the employees and the in-between coordination for project implementation.

A construction company can potentially take, according to its business model, any of the above types or their combination. The importance of each type for the organizational function itself and the interconnections among the constituent groups depends on each unique organizational case.

\subsection{Game Theory}

Game theory (GT) focuses on the interrelationship and interconnection of decisions made by different individuals or groups, where each one's decision affects the rest [20]. Specifically, it represents a systematic way of researching and investigating the following key aspects [20]: (a) the case study itself, noted as game, (b) the decision makers, noted as players or agents, (c) the interactions of the agents' decisions, (d) each agent's strategy, developed after the interpretation of the aforementioned interactions, and (e) the rationality of the agents' decisions (where it is factually considered that all agents will choose the most beneficial-for them-strategy). In real-life situations, agents tend to act irrationally (due to sentimental shifts, psychological patterns, peer pressure, and other reasons), which is partially explained with the adaptation of Kripke's theorem [21]. However, this is smoothed out when groups (and not individuals) are considered as single agents (not to 
be mistaken for coalitions of individual players), due to internal group processes during decision-making [22].

Cases with only one agent (single-person decisions) or too many of them (where each individual's decision effect upon the total system can be considered as infinitesimal) are not games and the approach of the Delphi method is more suitable [23]. However, apart from such extremes, extensive games with many agents can indeed exist, approached as lattices (with the nodes being the agents and the edges in between the interactions) and resolved following the survival-of-the-fittest rule and/or promoting agent cooperation [24,25].

There are various distinctive game types fitting a range of modelized problems, such as the competitive and potentially competitive zero-sum games, the cooperative and potentially cooperative non-zero-sum games (where Nash equilibria can exist), the dynamic or sequential games, the repetitive games, and the coalition games [20,26]. Notable game depictions are the strategic (matrixlike), the appositional, and the extendible [20].

Crucial in game formation is the concept of information, namely the system conditions and parameters (e.g., the number of agents and their available strategies) [20]. Relatively to how much of this information is known to the agents, games can be discretized as totalor short-information ones $[20,26]$. Short-information games should not be mistaken for the incomplete information sequential games, since incomplete information refers to the lack of knowledge concerning the previous-step agents' decisions and not to the general systemic framework [26].

Increasingly important regarding decision-making is evolutionary GT, namely the GT implementation in evolving populations [27]. Evolutionary GT focuses on the dynamics and frequency of strategy shifts in constantly changing agent systems [28]. Although affiliated with evolving populations, certain evolutionary GT research results can be applied to all kinds of games. A particularly important case regards conditional cooperation and states in which, when cooperative agents tend to agree in their opinion from the outset, a small number of competitive agents is actually beneficial to this cooperation, as they create (by the contradiction they impose) more time for the cooperative ones to properly reconsider their options and reevaluate their strategy-although too many competitive agents tend, indeed, to create confusing situations within the population [29].

Game theory is also used in conjunction with specified methodological frameworks of decision-making (such as the VIKOR method). Although the results of distinct implementation cases may vary, in certain models the two approaches can complement each other, resulting in unanimously admissible results [30].

\section{Modelling, Analysis and Results}

\subsection{Modelling of the Exemplar Reference Organization (Construction Company)}

The proposed methodological framework begins with the modelling of the construction company. This process involves two large categories of required information. The first concerns the unique typical features of the company, which are normally used in several classification schemes (e.g., size, activities, etc.). The second concerns the unique inherent organizational attributes and dynamics, which are determined by hard to trace and quantify factors (e.g., personal relations, human-equipment dependencies, etc.). Considering the insurmountable difficulties in collecting information in the second category and given the goal of this research, which is the initial conceptualization and verification of a methodological approach to minimize ostracism risk, it was decided not to adopt an approach of analysis based on the acquirement and processing of empirical data but, instead, consider and model an exemplar reference organization by following the first part of the "pattern marching" technique that qualitatively captures institutional logics-namely the conception of an "ideal type" [31]. This approach allows the description and modelling of an exemplar reference organization whose attributes correspond to the theoretical considerations in Section 2 and at the same time are informed by the one-dimensional view of power in [32] (since the conceptualization is embedded in the context of a single organization and concerns the decision-making interactions between agents in that specific context) and the 
considerations on properly modelling the dimensions of multi-agent organizations in [33]. To bring all the aforementioned elements together, as well as frame the synthesis of the constituent attributes of the exemplar reference organization, the paradigmatic approach of systems theory is used [34]. With systems theory, the constituents are identified as parts of a system, and their sum is taken to amount to less than the whole; therefore, an understanding of the context in which the conceptualization takes place (in the current case, the construction sector), is required [34].

Given the framing described above and the theoretical elements in Section 2, the exemplar reference organization (an "ideal type" construction company) is conceptualized to feature the following attributes:

- It will be an enterprise.

- Its legal form will be the one of S.A.

- It will be of medium size ( \pm 500 employees), avoiding the extreme market conditions associated with very large or small organizations. The rest of the competitive organizations are considered to be similarly sized.

- It will be private, avoiding practical restrictions inherent in public organizations (albeit the private market is itself strictly definite in terms of the appointed legal, associative, regulatory, conditional, and competitive framework).

- It will be a professional bureaucratic organization, with OC including, indicatively, engineers of various disciplines, construction managers, site managers, financial and administrative partners, technicians, and operative and labor workers. It will be relatively formalized but also decentralized vertically and horizontally to provide autonomy to OC professionals, and will use the standardization of skills as its prime coordinating mechanism [18]. The top management of its SA will be relatively small, and generally mutually cooperative with OC. However, in case of non-cooperation, there will be adverse effects for both SA and OC $[16,18]$. There will also be relatively few IL managers, generally oscillating between their support to SA and OC, and somewhat assuming a more powerful position when OC does not support SA [17]. TS will be restricted in size, with its support to the rest of the organizational groups mainly influenced by whether they disturb the procedural standardization it promotes [16]. Finally, SF will be typically large, providing clerical and maintenance support for OC and being largely influenced by it [18]. Despite its size, though, its own influence on the actions of the rest of the groups will be relatively weak [16-18].

- There will be no legal department, with any occurring instances being appointed to an external legal firm.

- It will abide organizationally to the Likert consultative model $([35,36]$, where there is partial communication (mainly of the consultative type) and group work in both the horizontal and the vertical hierarchical axes. Its motivation system will consist mainly of work reciprocation and the acknowledgement of the degree of involvement during the decision-making. However, in the said degree of involvement of the lower managerial levels, a more generous approach than the one of the classic consultative model is hereby attempted. Recent research on business economics has postulated that a stakeholder system of corporate governance relying on a cooperation between managers and employees has the potential to enhance long-term firm performance [37]. Particularly, it is considered that a robust employee representation on the board of directors, in an approximate $43 \%$ of the total number of the board participants, is beneficial [37]. This percentage is largely optimal [37], and although it emanated from the comparison between the allocation of the employees' representation and the Tobin's $Q$ coefficient (regarding, mainly, companies with vast share capital), it can be generalized and associated with all sorts of organizational performance indicators [37,38]. In praxis, the optimal percentage of $43 \%$ is not often found, but real case companies in e.g., Germany and Japan, are reportedly reserving more than a third of the board seats for employees' representatives [37]. This can lead to $43 \%$ being considered as achievable and in any case suitable for a conceptual modelling of an "ideal type" of 
organization, as is the case with the current study. Moreover, as such representatives can belong to various organizational groups and hierarchical levels [37], and following the denomination of the conceptualized construction company as a professional bureaucratic organization, it can be considered that most employee board members will belong to the OC, and fewer to the TS, IL, and SF. Additionally, the diffusion of responsibility among the employees is significant, due not only to the optimal representation percentage, but also to Organizational Citizenship Behavior (OCB), i.e., the behavior apparent in employees caring and striving for the collective organizational benefit [39] — where the employees will not only complete their appointed tasks in the most effective way, but also work beyond their formal professional obligations, for the benefit of the whole organization [40]. It should be noted, though, that the described consultative model is not generous to the degree that it can be generalized to the participatory model $[35,36]$.

- Its strategy will be both innovational and oriented to cost minimization, as aligned with strategy-oriented insights in $[4,7,41,42]$ for firms either explicitly identified as construction companies or largely having characteristics adjacent to the modelling attributes of the exemplar "ideal type" construction firm conceptualized here. The organizational structures will be medium-to-highly concentrated and the degree of control quite strict. Task discretization and the degree of standardization will be high-allowing, however, demarcated self-acting.

- The external market environment will be moderately stable, complex, and specific.

- Its structure will be amplitudinal, featuring high employee specialization, responsibility diffusion among the lower managerial levels, extensive control range, and fewer managerial levels [8].

- It will be, structurally, quite decentralized. Decision-making responsibilities will be diffused among the hierarchical ranks, and certain administrative nodes will be preferred instead of many intermediate managerial levels. Such decentralization is positively associated with the presence of lower managerial levels in the organizational structure, and indifferently or slightly negatively associated with the respective presence of higher managerial levels [36].

- Its most specialized employees, such as the engineers, will be able to organize into individual targeted groups undertaking certain aspects of a broader project [13].

- It will feature a distinguished and constantly updated information system, available to all employees [12]. The system will be partitioned into three distinctive information management stages, namely the exchange of information, its conception, and its use into creating value [43]. The criteria defining the partitioning are associated with the organizational Information and Communications Technology (ICT) background, its socio-technological dimension, its focus on lifecycle, and its perspective of generating and utilizing knowledge and information [43].

- Its corporate culture will promote neutrality, rationality, and minimized emotional and psychological interventions while deciding under risk, thus achieving the most effective approach to decision-making [44]. It will also favor cooperation, communication, and advantageous interaction among employees, as this improves the collaborative risk perception [45]. Finally, it will promote legitimate courses of action, rejecting immoral, parasitic, and rent-seeking behaviors, thus ensuring the absence of corruption in all internal and external organizational aspects [46].

- Employee training and knowledge updating will be continuous, brought about by specific quality consultants in accordance to the Total Quality principles [47].

- Its most specialized employees will be able to use wikis, BIM (Building Information Modelling), and other applications to optimize their cooperation, knowledge exchange and innovation [48]. The utilization of such applications requires clear usage purpose; collaborative culture; knowledge and information management capability; process standardization; and clear managerial intension [48]. 
Regarding the context, the synthesis (through a systems theory understanding) of the aforementioned attributes can be considered to represent the "ideal type" of a typical medium-sized construction company that could be found in a number of different construction sectors, despite the corresponding specificities of each sector-e.g., within the industry of the United States [49] or even developing countries [50].

The developed model can be generalized and is not restricted by special conditions, apart from those defined by the given theoretical framework.

\subsection{Non-Zero-Sum Game of the Organizational Decision-Making under Risk}

Having stated the characteristics of the exemplar organization, the GT modelling of its decision-making under risk will take place. This will be the first step for the calculation of the probability of organizational cooperation in the perspective of a decision under risk, considering both the maximization of individual, group and collective benefits and the minimization of the ostracism risk. A total of $N=5$ agents is considered, with each $i \in N$ representing a Mintzberg group: SA, OC, IL, TS, and SF. The main game attributes are the following:

- The agents interact through their strategic actions, and their choices are rational [20]. Rationality is empowered due to the agents being groups and not individuals [22].

- It will be a potentially cooperative, classic, finite, single-round, non-dynamic, nonsequential, non-repetitive, non-coalition, and non-zero-sum game [20,26], where Nash equilibria may exist but will not be highlighted, as this is not the purpose of the present modelling.

- It will be a complete information game, since all its conditions and parameters will be known and accessible to all agents. The context, framework, and transport of the said information are considered to not be influenced by common dilemmas [51].

- $\quad$ Resulting from OCB, agents are expected to try to seek the maximum total organizational benefit.

- The feasibility of cooperation among the agents generally depends on their number, individual benefits following their strategic choices, and intention to possibly change their strategy [52]. Since the present game includes a fixed agent number and is non-dynamic and non-sequential (i.e., the agents cannot change their strategy during the game), cooperation will mainly depend on the individual benefits. However, cooperation is favored by the adequate communication among the agents (which improves the risk perception), and the existence of institutional incentives (which promote OCB) [53].

- Any decisions concerning the organization are not only made by the SA, since employee representation is set to the optimal $43 \%$ of the board members [37].

The ascending agent benefit scale $P=\{1,2, \ldots, 9\}$ is defined, where 1 indicates the least possible satisfaction, 5 a medium satisfaction level (equal to the existing satisfaction prior the decision under risk), and 9 the maximum egoistic satisfaction. As far as the egoistic benefits are concerned, taking the decision under risk is more beneficial for the SA (since risk aversion is not considered to be a leading attribute and can culminate, in some cases, into organizational decline) [44]—whereas IL, TS, OC, and SF will mostly be medially satisfied, with OC and SF depicting a descending proclivity. Such satisfaction levels can be modified, depending on the strategic decisions of the agents (along with any concessions among them). For the definition of $P$, both quantitative (e.g., monetary profit or cost) and qualitative (e.g., working posterity and satisfaction in the workplace) criteria were considered, influencing both job performance and risk behavior.

The maximum egoistic benefit for each agent can appear when all other agents' benefits are lower than the said benefit. For example, SA will receive the maximum egoistic benefit if it imposes its opinion and makes the decision under risk without conceding towards the demands and claims of the other groups and, subsequently, of the whole organization. Such a behavior, though, violates the pursuance of OCB, which supports the conviction that individual benefits should imply the maximization of the organizational 
benefit. Nonetheless, OCB should not lead to injustice towards certain agents "for the common good".

Taking the aforementioned into consideration, it is necessary to connect all individual benefits to the single collective one and consider that the latter is only achieved when all agents receive at least a certain minimum but still sufficient level of satisfaction. For the present model, it is assumed that the minimum acceptable collective benefit sums up to 30 and is achieved when each of the 5 individual ones is at least equal to 6 on the $P$ scale (meaning that the corresponding agents are at least modestly benefited after the decisionmaking in an ascending manner). All interactions and correlations between the agents are defined in a way that no sum can be equal to 30 when there is even one individual benefit less than 6.

As far as the decision under risk itself is concerned, the agents have two options: to approve (AP) or disapprove (DSP) of it, thus favoring risk acceptance or aversion. Resulting is the set of strategies $S=\left\{s_{1}, s_{2}, \ldots, s_{m}\right\}, \mathrm{m}=2^{5}=32$. Each agent's dominant strategies (denoted as $\mathrm{Si}^{*}$ ) are considered the ones posing the maximum egoistical benefit. All strategies appear only once, but the benefit combination of some can be similar to that of others.

With the framework of the game outlined as above, it is possible to set the game itself with the strategic representation of Figure 2 (see next page).

\begin{tabular}{|c|c|c|}
\hline $\begin{array}{c}\text { OC } \\
\text { SA }\end{array}$ & AP & DSP \\
\hline AP & $7,7,7,7,7$ & $8,4,6,6,4$ \\
\hline DSP & $5,8,6,5,5$ & $5,5,5,5,4$ \\
\hline
\end{tabular}

IL: AP TS: AP SF: AP

\begin{tabular}{|c|c|c|}
\hline $\begin{array}{c}\text { OC } \\
\text { SA }\end{array}$ & AP & DSP \\
\hline AP & $7,7,7,6,8$ & $6,4,6,5,6$ \\
\hline DSP & $5,6,7,5,6$ & $6,7,5,6,5$ \\
\hline
\end{tabular}

IL: AP TS: DSP SF: AP

\begin{tabular}{|c|c|c|}
\hline $\begin{array}{c}\text { OC } \\
\text { SA }\end{array}$ & AP & DSP \\
\hline AP & $7,7,4,5,6$ & $5,7,6,6,4$ \\
\hline DSP & $7,5,6,6,3$ & $7,7,8,5,2$ \\
\hline
\end{tabular}

IL: DSP TS: DSP SF: AP

\begin{tabular}{|c|c|c|}
\hline $\begin{array}{c}\text { OC } \\
\text { SA }\end{array}$ & AP & DSP \\
\hline AP & $7,7,7,6,6$ & $6,4,6,5,3$ \\
\hline DSP & $7,4,5,7,5$ & $7,6,4,7,5$ \\
\hline
\end{tabular}

IL: AP TS: DSP SF: DSP

\begin{tabular}{|c|c|c|}
\hline $\begin{array}{c}\text { OC } \\
\text { SA }\end{array}$ & AP & DSP \\
\hline AP & $7,6,4,7,5$ & $7,4,5,7,5$ \\
\hline DSP & $6,4,6,5,3$, & $7,7,7,6,6$ \\
\hline
\end{tabular}

IL: DSP TS: AP SF: AP

\begin{tabular}{|c|c|c|}
\hline $\begin{array}{c}\text { OC } \\
\text { SA }\end{array}$ & AP & DSP \\
\hline AP & $7,7,8,5,2$ & $7,5,6,6,3$ \\
\hline DSP & $5,7,6,6,4$ & $7,7,4,5,6$ \\
\hline
\end{tabular}

IL: AP TS: AP SF: DSP

\begin{tabular}{|c|c|c|}
\hline $\begin{array}{c}\text { OC } \\
\text { SA }\end{array}$ & AP & DSP \\
\hline AP & $6,7,5,6,5$ & $5,6,7,5,6$ \\
\hline DSP & $6,4,6,5,6$ & $7,7,7,6,8$ \\
\hline
\end{tabular}

IL: DSP TS: AP SF: DSP

\begin{tabular}{|c|c|c|}
\hline $\begin{array}{c}\text { OC } \\
\text { SA }\end{array}$ & AP & DSP \\
\hline AP & $5,5,5,5,4$ & $5,8,6,5,5$ \\
\hline DSP & $8,4,6,6,4$ & $6,6,6,8,6$ \\
\hline
\end{tabular}

IL: DSP TS: DSP SF: DSP

Figure 2. Matrix representation of the modelled game for organizational groups in decision-making. 
The benefits are appointed to the corresponding agents relatively to the defined theoretical framework and the agents' expectations and incentives, and they are direct. For such an appointing, the following conceptual rules were followed through-with some flexibility, depending on the specificities of each case as described in the commentary that follows right after:

- Full cooperation on approval generates benefits greater than the medium level for all agents, which are largely equal.

- Full cooperation on disapproval generates benefits greater than the medium level for all agents, which, however, can be unequal relative to the strength and positioning of each agent.

- Disagreement between strong agents generally generates a high benefit for the ones cooperating with most of the rest of the agents, and a benefit around the medium level or slightly lower for the non-cooperative ones.

- Disagreement between weak agents generally generates a high benefit for the ones cooperating with most of the strongest agents, and a low benefit for the ones siding with other relatively weak agents.

- Isolation of strong agents results in corresponding benefits around the medium level or slightly lower. The benefits for the rest of the agents depend not only on their clustered cooperation, but also their relative position to the isolated strong agent.

- Isolation of weak agents results in severely low corresponding benefits. The benefits for the rest of the agents depend mainly on their clustered cooperation, and only partially on their relative position to the isolated weak agent.

In time, the in-organization correlations and, consequently, the corresponding benefits, may change. This is increasingly important when it comes to large egoistic benefits of certain agents (and the corresponding minimized benefits of others), because in such cases there may be long-term skirmishes and degraded situations among them, leading to loss even for the groups that were initially (and egoistically) largely benefited.

In the following, a commentary on each of the possibly chosen strategies (considering the agent order SA, OC, IL, TS, and SF in the delineation of benefits) will take place.

\subsubsection{Case $\mathrm{S}_{1}=\{\mathrm{AP}, \mathrm{AP}, \mathrm{AP}, \mathrm{AP}, \mathrm{AP}\}=(7,7,7,7,7)$ (sum: 35$)$}

When everyone agrees to AP, there is full cooperation. For this to happen, SA will have to bestow privileges upon the other agents, thus somewhat reducing its individual benefit (which is still large). OC, being the paramount group, is equally benefited, as well as IL and SF, which are similarly interconnected with SA and OC (SF is closer to OC and IL closer to SA). TS is also equally benefited, even though a decision under risk could be destabilizing, to some extent, to the organizational prototyping implemented by its analysts.

\subsubsection{Case $\mathrm{S}_{2}=\{\mathrm{AP}, \mathrm{DSP}, \mathrm{AP}, \mathrm{AP}, \mathrm{AP}\}=(8,4,6,6,4)$ (sum: 28$)$}

When $\mathrm{OC}$ disapproves and everyone else approves, its benefit collapses under the medium level of satisfaction. SF is drifted along OC, in spite of agreeing with the rest, because it is strongly interconnected with OC. The direct egoistical benefit of SA is large, since it has no interest in earning the cooperation of OC and does not bestow any privileges upon it. IL earns a modest benefit, not being too large due to the non-cooperation of OC. TS is granted an equally modest benefit and is not directly affected by the reluctance of OC.

\subsubsection{Cases $\mathrm{S}_{3}=\{\mathrm{DSP}, \mathrm{AP}, \mathrm{AP}, \mathrm{AP}, \mathrm{AP}\}=(5,8,6,5,5)$ and $\mathrm{S}_{30}=\{\mathrm{AP}, \mathrm{DSP}, \mathrm{DSP}, \mathrm{DSP}, \mathrm{DSP}\}=$} $(5,8,6,5,5)$ (sum: 29)

When SA is solely disagreeing with the rest of the agents, its benefit is only medium, but not lower in any case. OC acts as the main pressure group, enforcing its opinion, and is largely benefited. IL takes advantage of the pressure imposed by the OC and is also benefited. However, its benefit is not as large, because it is also closely interconnected to SA. With SA and OC being in adversity, SF and TC are largely caught in the middle and their benefits are mediocre. 
3.2.4. Cases $\mathrm{S}_{4}=\{\mathrm{DSP}, \mathrm{DSP}, \mathrm{AP}, \mathrm{AP}, \mathrm{AP}\}=(5,5,5,5,4)$ and $\mathrm{S}_{29}=\{\mathrm{AP}, \mathrm{AP}, \mathrm{DSP}, \mathrm{DSP}, \mathrm{DSP}\}=$ $(5,5,5,5,4)$ (sum: 24$)$

When SA and OC agree and the rest disagree with them, then their individual benefits are mediocre. Their mutual opinions and benefits are strongly influential to the benefits of the disagreeing agents and reduce them around the medium level as well. Albeit, SF is the one that suffers the greatest loss, as it is heavily influenced by both SA and OC (mainly OC).

3.2.5. Cases $\mathrm{S}_{5}=\{\mathrm{AP}, \mathrm{AP}, \mathrm{DSP}, \mathrm{AP}, \mathrm{AP}\}=(7,6,4,7,5)$ and $\mathrm{S}_{28}=\{\mathrm{DSP}, \mathrm{DSP}, \mathrm{AP}, \mathrm{DSP}, \mathrm{DSP}\}=$ $(7,6,4,7,5)$ (sum: 29)

When IL disagrees with the rest, it suffers significant loss. The largest benefit is equally granted to SA and TS, with SA not surpassing TS because it lacks support on the part of IL. TS is instead primed for the same reason. OC is modestly benefited, as it is influenced by its disagreement with IL. SF is not directly influenced by the relative commotion and its benefit sticks to the medium level.

3.2.6. Cases $\mathrm{S}_{6}=\{\mathrm{AP}, \mathrm{DSP}, \mathrm{DSP}, \mathrm{AP}, \mathrm{AP}\}=(7,4,5,7,5)$ and $\mathrm{S}_{27}=\{\mathrm{DSP}, \mathrm{AP}, \mathrm{AP}, \mathrm{DSP}, \mathrm{DSP}\}=$ $(7,4,5,7,5)$ (sum: 28$)$

In these cases, TS is equally benefited to SA. This happens because, not being supported by either OC or IL, SA is forced to bestow considerable privileges on TS to earn its support. The benefit of OC is greatly decreased, and the one of IL is medium because it is similarly interconnected to SA and OC. SF, while agreeing with SA and TS, is also granted with a medium benefit, because it is heavily influenced by the disagreeing OC.

3.2.7. Cases $\mathrm{S}_{7}=\{\mathrm{DSP}, \mathrm{AP}, \mathrm{DSP}, \mathrm{AP}, \mathrm{AP}\}=(6,4,6,5,3)$ and $\mathrm{S}_{26}=\{\mathrm{AP}, \mathrm{DSP}, \mathrm{AP}, \mathrm{DSP}, \mathrm{DSP}\}=$ $(6,4,6,5,3)$ (sum: 24$)$

The agreement between OC, TS, and SF is not highly beneficial for them, since OC, although strongly influential, has only managed to side with the two less dominant groups. The situation is extremely unfavorable to SF, largely unfavorable to OC, and mediocrely favorable to TS, which is more resistant to benefit ups-and-downs. The benefits of SA and $\mathrm{IL}$, favored by their cooperation, are modestly increased.

3.2.8. Cases $\mathrm{S}_{8}=\{\mathrm{DSP}, \mathrm{DSP}, \mathrm{DSP}, \mathrm{AP}, \mathrm{AP}\}=(7,7,7,6,6)$ and $\mathrm{S}_{25}=\{\mathrm{AP}, \mathrm{AP}, \mathrm{AP}, \mathrm{DSP}, \mathrm{DSP}\}=$ $(7,7,7,6,6)$ (sum: 33 )

When SA, OC, and IL agree, they form a strong cooperative cluster and are equally granted with increased benefits. TS and SF, although both disagreeing with the above agents, are also modestly benefited instead of suffering avail losses, because both OC and IL partly prime them.

3.2.9. Cases $\mathrm{S}_{9}=\{\mathrm{AP}, \mathrm{AP}, \mathrm{AP}, \mathrm{DSP}, \mathrm{AP}\}=(7,7,7,6,8)$ and $\mathrm{S}_{24}=\{\mathrm{DSP}, \mathrm{DSP}, \mathrm{DSP}, \mathrm{AP}, \mathrm{DSP}\}=$ $(7,7,7,6,8)$ (sum: 35$)$

The cooperative cluster of the immediately prior cases is furtherly reinforced by SF. The benefit of SF is the largest among the four agreeing agents, since SF favors and is favored by the three more dominant agents and opposes TS, which is opposed to the loose structure typically favored by SF.

3.2.10. Cases $\mathrm{S}_{10}=\{\mathrm{AP}, \mathrm{DSP}, \mathrm{AP}, \mathrm{DSP}, \mathrm{AP}\}=(6,4,6,5,6)$ and $\mathrm{S}_{23}=\{\mathrm{DSP}, \mathrm{AP}, \mathrm{DSP}, \mathrm{AP}, \mathrm{DSP}\}=$ $(6,4,6,5,6)$ (sum: 27$)$

In these cases, SA cooperates with IL and SF. However, without the support of OC, their benefits are only modestly increased. OC, on the other hand, suffers significant decrease to its benefit, due to its opposition to SA and IL, and its cooperation only with TS. TS itself experiences no benefit variance. 
3.2.11. Cases $\mathrm{S}_{11}=\{\mathrm{DSP}, \mathrm{AP}, \mathrm{AP}, \mathrm{DSP}, \mathrm{AP}\}=(5,6,7,5,6)$ and $\mathrm{S}_{22}=\{\mathrm{AP}, \mathrm{DSP}, \mathrm{DSP}, \mathrm{AP}, \mathrm{DSP}\}=$ $(5,6,7,5,6)$ (sum: 29$)$

Since IL opposes SA, it assumes a somewhat leading role in the cooperative cluster among itself, OC and SF-although OC remains the most influential agent. This informal leadership grants IL with increased benefit, whereas the rest of the three cooperating agents are modestly benefited. SA, on the other hand, is stuck in the medium level of satisfaction. So is TS, mainly because a less dominant and strict TS individually favors the empowered and cooperating IL and SF.

3.2.12. Cases $\mathrm{S}_{12}=\{\mathrm{DSP}, \mathrm{DSP}, \mathrm{AP}, \mathrm{DSP}, \mathrm{AP}\}=(6,7,5,6,5)$ and $\mathrm{S}_{21}=\{\mathrm{AP}, \mathrm{AP}, \mathrm{DSP}, \mathrm{AP}, \mathrm{DSP}\}=$ $(6,7,5,6,5)$ (sum: 29)

In these cases, $\mathrm{OC}$ is able to impose its opinion to the agents it cooperates with and be granted with increased benefit. SA and TS also benefit, but only modestly. The coalition of IL and SF, on the other hand, can prevent heavy benefit losses, but their opposition to the more dominant $\mathrm{OC}$ and SA also keeps their benefits around the medium level.

3.2.13. Cases $\mathrm{S}_{13}=\{\mathrm{AP}, \mathrm{AP}, \mathrm{AP}, \mathrm{AP}, \mathrm{DSP}\}=(7,7,8,5,2)$ and $\mathrm{S}_{20}=\{\mathrm{DSP}, \mathrm{DSP}, \mathrm{DSP}, \mathrm{DSP}, \mathrm{AP}\}=$ $(7,7,8,5,2)$ (sum: 29)

When SF is solely disagreeing with the rest, its benefit is decreased closely to the utmost minimum, as it is the weakest agent. IL imposes its demands (partly to counterbalance the defection of SF, however weak it may be) over SA and OC and benefits even more than SA and OC. TS, although being a member of the four-part cooperative cluster, is negatively affected by the imposition of IL and its benefit is mediocre.

3.2.14. Cases $\mathrm{S}_{14}=\{\mathrm{AP}, \mathrm{DSP}, \mathrm{AP}, \mathrm{AP}, \mathrm{DSP}\}=(7,5,6,6,3)$ and $\mathrm{S}_{19}=\{\mathrm{DSP}, \mathrm{AP}, \mathrm{DSP}, \mathrm{DSP}, \mathrm{AP}\}=$ $(7,5,6,6,3)$ (sum: 27$)$

$\mathrm{OC}$, cooperating only with $\mathrm{SF}$, does not benefit. SF is severely affected by its opposition to SA, IL, and TS, and its benefit is only slightly increased relative to the immediately prior cases, due to the support of OC. Without the cooperative influence of OC, SA imposes its opinion upon IL and TS, and its benefit surpasses theirs.

3.2.15. Cases $\mathrm{S}_{15}=\{\mathrm{DSP}, \mathrm{AP}, \mathrm{AP}, \mathrm{AP}, \mathrm{DSP}\}=(5,7,6,6,4)$ and $\mathrm{S}_{18}=\{\mathrm{AP}, \mathrm{DSP}, \mathrm{DSP}, \mathrm{DSP}, \mathrm{AP}\}=$ $(5,7,6,6,4)$ (sum: 28$)$

OC is relatively imposing itself over IL and TS, its benefit surpassing the modest ones of the other two. SA is only mediocrely benefited, and the benefit of SF is below the medium level, being slightly increased relative to the immediately prior cases, due to the support of SA. This increase, however, is not that significant, since SF is opposing OC.

3.2.16. Cases $\mathrm{S}_{16}=\{\mathrm{DSP}, \mathrm{DSP}, \mathrm{AP}, \mathrm{AP}, \mathrm{DSP}\}=(7,7,4,5,6)$ and $\mathrm{S}_{17}=\{\mathrm{AP}, \mathrm{AP}, \mathrm{DSP}, \mathrm{DSP}, \mathrm{AP}\}=$ $(7,7,4,5,6)$ (sum: 29$)$

SA and OC are largely benefited. SF is only modestly benefited, because although it cooperates with SA and OC, its influence is relatively weak regarding its benefit demands. The benefit of TS remains stagnant and the one of IL is decreased, since it opposes SF and, principally, OC and SA. Cooperation between TS and IL gives rather poor results, since the interconnection between them is weaker than the one between other agents (e.g., between SA and IL).

\subsubsection{Case $\mathrm{S}_{31}=\{\mathrm{DSP}, \mathrm{AP}, \mathrm{DSP}, \mathrm{DSP}, \mathrm{DSP}\}=(7,4,6,6,4)$ (sum: 27$)$}

This case is quite similar to $S_{2}=\{\mathrm{AP}, \mathrm{DSP}, \mathrm{AP}, \mathrm{AP}, \mathrm{AP}\}$. The benefits are similar, too, except for the one granted to $\mathrm{SA}$, which is slightly lower than the corresponding one in $S_{2}$. This is due to the risk aversion highlighted in the disapproval of the decision under risk, which can possibly lead to a waste of profit opportunity, primarily affecting SA. 


\subsubsection{Case $\mathrm{S}_{32}=\{\mathrm{DSP}, \mathrm{DSP}, \mathrm{DSP}, \mathrm{DSP}, \mathrm{DSP}\}=(6,6,6,8,6)$ (sum: 32$)$}

In the universal disapproval of the decision under risk, there is full cooperation, such as $S_{1}$. However, the individual benefits are different. SA, OC, IL, and SF are only modestly benefited, whereas the benefit of TS is largely increased, because of not only the successful cooperation with the rest of the agents but also of the unanimous risk aversion, which means-among other things - that the standard and modelled organizational structures will probably remain fundamentally unchanged.

\subsubsection{Discussion on the Case Commentary}

Seconding the delineated justification of the model results, the following point can be made:

- The agents' dominant strategies are, respectively, $S^{*} \mathrm{SA}=S_{2}=(8,4,6,6,4)$ (sum: 28$)$, $S^{*}{ }_{\mathrm{OC}}=S_{3}=S_{30}=(5,8,6,5,5)$ (sum: 29), $S^{*} \mathrm{IL}=S_{13}=S_{20}=(7,7,8,5,2)$ (sum: 29), $S^{*} \mathrm{TS}=S_{32}=(6,6,6,8,6)$ (sum: 32$)$ and $S^{*}{ }_{\mathrm{SF}}=S_{9}=S_{24}=(7,7,7,6,8)$ (sum: 35$)$. The ones of SA, OC, and IL entail that there will be certain agents with no satisfactory benefits. On the contrary, the ones of TS and SF include benefits $\geq 6$ on the benefit scale $P$, where 6 is the minimum acceptable individual benefit. Only $S^{*} \mathrm{TS}$ and $S^{*} \mathrm{SF}$ sum up to more than 30 (the minimum defined collective benefit), and thus only these 2 are included in the set of the preferable cooperative strategies processed in the methodology's next application step. The less favoring of cooperation when SA, OC, and IL are respectively dominant emanates from the power and influence of those groups (with OC being the most prominent and accommodating professionals potentially ranging from designers and structural engineers to supply chain facilitators and site managers). When any of those is dominant, its power largely cannot be cooperatively controlled by the rest. On the other hand, TS and SF, much less influential, become dominant only when the rest of the agents are at least modestly benefited.

- The extreme ends of $P, 1$, and 9, did not appear in any case. It was considered that such individual benefits of even the less prominent agents, are not in line with certain attributes of the exemplar reference organization-namely, its generous Likert consultative model, decentralized and amplitudinal structure, elements of its organizational culture, and OCB. Such extremities granting immediate benefits but being unsustainable in the long run for the described "ideal type" construction company can be, in the case of e.g., the SA, an extreme form of strategic retrenchment in the face of the COVID-19 pandemic [41].

- $\quad$ SA can never be granted with a benefit less than the mediocre 5. Representing a total $57 \%$ of the participants in the board of directors, it is highly unlikely that it would allow decisions that it considers too unfavorable (e.g., relinquishing power to the middle managers of IL or the site managers of OC). In most cases where SA is isolated or sides only with TS and/or SF, it is mediocrely benefited. In all the rest, it is generally benefited at least modestly.

- OC, being the paramount group, is greatly influential in cooperative clusters including three or more agents, where its individual benefit is increased, and the rest of the cooperating agents are benefited at least modestly. OC influences SF in a way preventing it from being satisfactorily, or even mediocrely, benefited, when they do not cooperate. The highest non-egoistic benefits of OC appear when it cooperates at least with SA and/or IL (thus aligning itself with upper and/or middle management). Such a cooperation can be achieved in, e.g., the case of innovation investments for updating the digital technologies used in construction planning. When isolated, it is never granted with less than 4 on the $P$ scale.

- IL is equally influenced by SA and OC and its benefit is linked to theirs. There are cases where a non-beneficial for SA situation empowers IL, which assumes profound hierarchical power, and others where the loss of SA leads to similar loss for IL, due to their failed cooperation. This flexibility in the positioning of IL between SA and OC in construction companies can be considered to reflect the mediating role assumed by the 
respective middle managers in several contexts, e.g., in Denmark and the UK [42], or Sweden [7]. When it comes to the rest of the Mintzberg groups, any empowerment of TS is not considered favorable towards IL, because TS largely seeks to impose machine bureaucratic organizational procedures, possibly removing privileges from the IL operators. SF does not generally affect IL.

- $\mathrm{TS}$, following the attributes of its default role, is largely benefited around the medium level, with little diversification. It is generally more benefited when participating in DSP-oriented cooperative clusters, due to the cooperation itself and, also, to the risk aversion inherent in DSP. Such risk aversion is generally translated into little or no alteration of the standardized procedures favored by TS. In private rather than public entrepreneurial organizations, such as the currently modelled construction company, TS is generally not as dominant; for example, see the study on Swedish site managers retaining their professional freedom and independence against standardization procedures being implemented during production [54]. As such, TS does not significantly perturb the equilibria among the other organizational groups.

- $\mathrm{SF}$, although greater in terms of representation relatively to TS, is considered the weakest group in a professional bureaucratic organization, something also affecting its benefits. When isolated, it is meagerly benefited. In most cases, OC strongly and positively influences SF. On the other hand, SF is generally benefited inversely to IL. SA is also greatly influential to SF, although less than OC and IL. TS is mostly neutral to SF, although slight TS benefit increases can lead to slight SF benefit decreases and vice versa.

- When cooperative clusters are formed and immediate benefits are at least modest, it is considered that medium- and long-term benefits will also be satisfactory. On the other hand, groups initially benefited too favorably or too severely are considered to cause destabilizing medium- and long-term situations due to, respectively, the protest of the rest against them for being too benefited, or their own protest when being too underprivileged; however, the rate of entropy caused by a group is proportional to its influence.

- Extended or full cooperation between, at least, the most empowered of the agents (e.g., in the case of large project portfolios or in order to support joint ventures with other construction and/or engineering companies), resulted in overall satisfactory individual and collective benefits. On the contrary, disagreements and dispersion of views were mostly considered to result in egoistic benefits and overall low levels of satisfaction.

- The increased individual benefits and the subsequent large collective benefit in cooperation cases are in order with the organizational approach favoring OCB. Such an approach does not exclude possible egoistic behaviors from time to time; however, in its framework the cooperative agents choose their course of action with seldom egoistic outbreaks.

Having shown the way to analyze the roles and behaviors of the various agents in decision-making in an organization, the probabilistic processing with the use of the bounded Pareto distribution follows.

\subsection{Probabilistic Processing of the Model and Final Results}

The external risk can be translated into monetary profit or loss, betterment or tarnishing of corporate reputation, and so on. However, it is the ostracism risk, accruing from the benefit allocation in the game set earlier, that will be processed probabilistically in the current section. The ostracism risk can result in short-, medium-, and long-term destabilizing situations inside the organization, caused by the dissatisfaction of the most underprivileged agents due to unfavorable benefit bestowal during the decision-making. Such unfavorable benefit allocation mainly occurs when a group is isolated and denies or is denied cooperation with at least some of the rest of the groups. Lack of cooperation, dispersion of views and large benefit diversity during the decision-making can lead to severe 
ostracism risk, no matter whether the decision proves beneficial or damaging regarding the external risk. It is presupposed that all agents are fully responsible for their choices, without responsibility withdrawal on their end, should the outcome of their decisions prove damaging.

Important to cooperative decision-making is the probability of such a cooperation. Although each strategy appears 1 in 32 times in the devised model (despite the individual and collective benefits being 2 by 2 identical in most cases), the probability of decisionmaking with a collective benefit summing up at least to 30 , follows a power law probability distribution - and, particularly, the bounded Pareto distribution.

The Pareto principle (also known as the 80-20 rule) states that in many cases, grossly $80 \%$ of the effects result from $20 \%$ of the causes [55]. It is widely used in numerous scientific fields and has been empirically observed as suitable for a wide range of natural, social, financial [56], and entrepreneurial [55,57] phenomena. Mathematically, the $80-20$ rule is a special case of the Pareto power law distribution. It is a rule: (a) persistent, as $80 \%$ of the previous $80 \%$ of the effects is the result of $20 \%$ of the previous $20 \%$ of the causes [55], and (b) symmetric, meaning that if $80 \%$ of the effects come from $20 \%$ of the causes, then the remaining $20 \%$ of the effects comes from the remaining $80 \%$ of the causes [55]. Being an illustration of a power law relationship and due to its self-similarity [58] over an expanded magnitude range, it produces radically different outcomes from Gaussian distribution phenomena [59]. Many sophisticated organizational, social, and financial models based on and around the Gaussian distribution experience frequent breakdowns [60], whereas the Pareto distribution is much more suitable [59]. The current model deals with the ostracism risk, and therefore the use of the Pareto distribution is well justified.

In the case generally described by the ordinary Pareto distribution (type I), the probability that the random variable $X$ is greater than a given number $X$ is calculated by the respective survival function [59]. When the random variable $X$ is demarcated in both ends (as in the current model), meeting not only a lower limit $X_{m}>0$ (the minimum possible value of $X)$, but also an upper limit $X_{u}\left(0<X_{m} \leq X \leq X_{u}\right)$, the special case of the bounded Pareto distribution, as in Equation (1), should be used [59]:

$$
\bar{F}(x)=\operatorname{Pr}\left(x<X \leq x_{u}\right)=\left\{\begin{array}{c}
\frac{x_{m}^{\alpha} \cdot x^{-\alpha}-\left(\frac{x_{m}}{x_{u}}\right)^{\alpha}}{1-\left(\frac{x_{m}}{x_{u}}\right)^{\alpha}}, x_{m} \leq x \leq x_{u} \\
1, x<x_{m} \& x>x_{u}
\end{array}\right\}
$$

where $\alpha \in \mathfrak{R}$ is the scale parameter known as the Pareto index.

In the current modelling case, the lowermost limit is $X_{m}=24$ (the collective benefits of $S_{4}, S_{7}, S_{26}$, and $S_{29}$ ), the upper limit is $X_{u}=35$ (the collective benefits of $S_{1}, S_{9}$ and $S_{24}$ ), and the base value is $X=30$ (corresponding to a conditional benefit case of $(6,6,6,6,6)$ ). As noted in the previous section, all interactions among the agents are defined so as no collective benefit can be at least equal to 30 when there is even 1 individual benefit that is less than 6 on the $P$ scale. The Pareto index is defined as $\alpha=\log _{4}(5)=1.161$, as derived from the Lorenz curve formula $L(F)$ resolved for the 80-20 rule percentages [59,61].

Considering the above, the probability of a collective organizational benefit $\geq 30$ which is, therefore, close to the optimal $80 \%$ of the maximum benefit (corresponding to the sum of the extreme conditional benefit case $(9,9,9,9,9))$, is calculated in Equation (2):

$$
\bar{F}(x)=\operatorname{Pr}(30<X \leq 35)=\frac{24^{1.161} \cdot 30^{-1.161}-\left(\frac{24}{35}\right)^{1.161}}{1-\left(\frac{24}{35}\right)^{1.161}}=0.35655 \approx 35.66 \%
$$

This means that although the cooperative strategic cases are proportionally less than $20 \%$ of the total amount, the possibility that such a case will happen is $35.66 \%$.

Sticking to the 80-20 rule and $\alpha=1.161$, when changing $X_{m}, X$, and $X_{u}$, respectively, the above results will also change. Certain computational tests were conducted for the probability trends to be ascertained: 
- When all three $X_{m}, X$, and $X_{u}$ increase (decrease), $\bar{F}(X)$ increases (decreases) as well.

- When $X_{m}$ increases (decreases) and $X, X_{u}$ remain stable, $\bar{F}(X)$ increases (decreases) as well. If the lower limit increases to the point where it is equal to $X$, then $\bar{F}(X)=100 \%$. On the contrary, if it decreases to $5 \times 1=5$ of the conditional benefit case $(1,1,1,1,1)$, then $\bar{F}(X)<2.3 \%$.

- When $X_{u}$ increases (decreases) and $X_{m}, X$, remain stable, $\bar{F}(X)$ increases (decreases) as well. If the upper limit decreases to be equal to $X$, then $\bar{F}(X)=0 \%$. On the contrary, if it increases up to $5 \times 9=45$ of the conditional benefit case $(9,9,9,9,9)$, then $\bar{F}(X)>55 \%$.

- When $X$ increases (decreases) and $X_{m}, X_{u}$ remain stable, $\bar{F}(X)$ inversely decreases (increases). If $X=X_{m}$, then $\bar{F}(X)=100 \%$. On the contrary, if $X=X_{u}$, then $\bar{F}(X)=0 \%$.

- When two out of three indexes are similarly varied and the third remains stable or is varied inversely to them, no general rule for the probability trends can be manifested, and each case should be tested individually.

The most benefited agent in the field of optimal cases is indeed OC, a result consistent with a professional bureaucratic organization following a generous consultant Likert model. In the rest of the cases, OC is variously benefited, but still influences greatly the rest of the agents' benefits.

\section{Discussion and Conclusions}

This paper investigates methodologically the probability of organizational cooperation during decision-making under risk, considering the maximization of individual, group and collective benefits, and the minimization of the, hereby introduced, ostracism risk. The ostracism risk is defined as the risk accruing from the lack of satisfaction of possibly underprivileged constituent personnel components during the process of the decisionmaking. For the current investigation, an exemplar reference organization (in the form of an "ideal type" private construction company) was modelled via game theory, with its organizational aspects emanating from organizational and HRM theories. The results taken from the said modelling was probabilistically processed using the bounded Pareto distribution. Thus, the probability of the adoption of a favorable and cooperational solution was estimated.

The proposed model is, up to this point, the only found in the relevant literature that incorporates a probabilistic process of the results taken from a devised game theory analysis of the ostracism risk. Specifically, the model measures the probability of decision-making that generally favors both individual and collective organizational benefits. The principal finding of the model is that such a satisfactory outcome is quite probable if the constituent organizational groups largely cooperate and assume full responsibility of their choices. Both preconditions meet the requirements of current organizational and HRM theorieshowever, such findings and preconditions do show implications when it comes to their relation to previous research. The studied literature has not included any succinct findings of tangible relations (in the form of quantified results) between the existence or lack of the ostracism risk and organizational effects. While this might be expected for a notion (i.e., "ostracism risk") that is introduced for the first time in this context (i.e., organizational theory with application in the construction industry), other research efforts on similar topics that were reviewed also lacked such evidence. Nonetheless, conceptual and methodological models-by nature-do not apply to specific conditions but represent general structures aiming at providing insights and handling tools for dealing with specific issues of interest. As such, by choosing a well-established and concretely validated organizational model (i.e., Mintzberg's) that generally applies to all types of organizations (including construction companies) as the basis for the proposed methodological framework, it can be considered that the current study can be well-contextualized in the relevant themes of previous research.

Given the study's methodological and theoretical framework, as well as the conceptualization and modelling of the exemplar reference construction company (of an "ideal type" ${ }^{\prime \prime}$, it can be considered that the proposed approach, upon application, can potentially 
benefit a construction firm featuring attributes similar or adjacent to those of the modelled reference company-i.e., a private, for-profit medium-sized organization, mainly based on its operating core. While the conceptual characteristics described within the present paper are, in themselves, ideal (as the "ideal type" characterization implies), a large number of real construction companies can broadly approach those characteristics in several real contexts. As an example of a case in point, in the context of Sweden, the vast majority of the 177,367 enterprises in the broad construction sector (as of 2019) are considered to be private SMEs [62], a subset of which are medium-sized companies. As such, in this context and other similar contexts, there is a large potential that the results of the current study can resonate with and provide benefits to a plethora of construction firms.

When it comes to what such benefits actually entail, it is clearly derived from the methodological and theoretical conceptions of the current effort that cooperation in decisionmaking across organizational groups and levels throughout the corporate structure is beneficial-also for the case of construction companies. This study verifies a methodological framework that upon conclusion of the steps required for improvements and validation will apply to any construction company to support and promote the utility of such a cooperation through its game theoretic and probabilistic modelling, which strives towards the minimization of the so-called "ostracism risk". As such, it can be considered that the proposed methodology can provide benefits to company strategists with regard to the planning of group decision-making processes, as well as the devising of corporate strategic responses to critical situations under risk. For example, a company's strategic responses against the COVID-19 pandemic crisis are considered in [41] to either reflect retrenchment, perseverance, innovation, or exit—all of which are crucially affected by factors related to (group) decision-making.

The limitations of the current study mainly point to the way the 9-point benefit scale $P$ was defined to depict the satisfaction levels of the game agents during the decisionmaking under risk, as well as the modelling following an "ideal type" conceptualization not corroborated by real data.

When it comes to the benefit scale, the benefit levels are in line with the defined theoretical framework, consider both quantitative and qualitative criteria, and were appointed after detailed deductions; however, a more robust scale definition by utilizing actual organizational databases, bibliographical examples, and/or case studies (e.g., interconnections between certain decisions of the board of directors and the amount of mediumand long-term employee complaints), can be proposed as future work. Nonetheless, such organizational databases are hard to find. Moreover, it should be noted that in the relative research field, the corresponding procedure in scale definitions is quite arbitrary and the justification rudimentary.

When it comes to the modelling, certain improvements can be recommended as future work, such as: (a) the creation of organizational databases, concerning past events in real-life enterprises, to be quantified and used as input data for the validation of the model, (b) the utilization of unexploited game theory elements, such as the iterated elimination of dominated strategies (IEDS), possible Nash equilibria, and utility measures, for the production of secondary but still useful results, (c) the utilization of other game types, such as the dynamic or sequential games (rounds of negotiations between the agents, or cost parleys), (d) the calibration and adaptation of $P$, processing statistical data (e.g., employee preferences and aspirations, or other relative organizational and corporate aspects) with utility functions, or by combining game theory with real options analysis (ROA) and AHP (Analytical Hierarchy Process), (e) the modification of the minimum acceptable collective benefit by including individual benefits that may not be even modestly satisfactory, and (f) possibly utilizing different probability distribution functions.

The model can be generalized and applied on all kinds of construction companies, provided that the organizational parameters and, particularly, the group decision-making process are properly defined. The use of game theory depends on the basic assumptions of the rest of the utilized theoretical frameworks. In the generalized case, certain game 
theory aspects (such as the number of the agents and the appointed benefits) are modified according to the initial theoretical framework of the model. The validation of the proposed methodology can be investigated through the application of the same evaluation criteria that apply to known group decision-making techniques. Examples of such criteria are applicability, efficiency, fairness, member satisfaction and morale, and leadership effectiveness.

Author Contributions: Conceptualization, D.K. and Y.X.; methodology, D.K.; validation, D.K.; formal analysis, D.K.; investigation, D.K. and Y.X.; resources, D.K. and Y.X.; data curation, D.K.; writingoriginal draft preparation, D.K. and Y.X.; writing-review and editing, D.K. and Y.X.; visualization, D.K.; supervision, Y.X.; project administration, Y.X. All authors have read and agreed to the published version of the manuscript.

Funding: This research received no external funding.

Conflicts of Interest: The authors declare no conflict of interest.

\section{References}

1. Meidell, A.; Kaarbøe, K. How the enterprise risk management function influences decision-making in the organization-A field study of a large, global oil and gas company. Br. Account. Rev. 2020, 49, 39-55. [CrossRef]

2. Hoskisson, R.E.; Chirico, F.; Zyung, J.; Gambeta, E. Managerial risk taking: A multitheoretical review and future research agenda. J. Manag. 2017, 43, 137-169. [CrossRef]

3. Asrar-ul-Haq, M.; Kuchinke, K.P.; Iqbal, A. The relationship between corporate social responsibility, job satisfaction, and organizational commitment: Case of Pakistani higher education. J. Clean. Prod. 2017, 142, 2352-2363. [CrossRef]

4. Wach, B.A.; Wehner, M.C.; Weißenberger, B.E.; Kabst, R. United we stand: HR and line managers' shared views on HR strategic integration. Eur. Manag. J. 2020. [CrossRef]

5. Adler, B.-M.; Baets, W.; König, R. A complexity perspective on collaborative decision making in organizations: The ecology of group-performance. Inf. Manag. 2011, 48, 157-165. [CrossRef]

6. Lin, J.-R.; Wu, D.-P. An approach to twinning and mining collaborative network of construction projects. Autom. Constr. 2021, 125. [CrossRef]

7. Simu, K.; Lidelöw, H. Middle managers' perceptions of operations strategies at construction contractors. Constr. Manag. Econ. 2019, 37, 351-366. [CrossRef]

8. Daft, R.L.; Armstrong, A. Organization Theory and Design; Nelson Education: Toronto, ON, Canada, 2012.

9. Amui, L.B.L.; Jabbour, C.J.C.; Jabbour, A.B.L.D.S.; Kannan, D. Sustainability as a dynamic organizational capability: A systematic review and a future agenda toward a sustainable transition. J. Clean. Prod. 2017, 142, 308-322. [CrossRef]

10. Daniels, S.R.; Wang, G.; Lawong, D.; Ferris, G.R. Collective assessment of the human resources management field: Meta-analytic needs and theory development prospects for the future. Hum. Resour. Manag. Rev. 2017, 27, 8-25. [CrossRef]

11. Armstrong, M.; Taylor, S. Armstrong's Handbook of Human Resource Management Practice, 14th ed.; Kogan Page Publishers: London, UK, 2017.

12. Chakraborty, A.R.; Mansor, N.N.A. Adoption of Human Resources Information System: A Theoretical Analysis. Procedia Soc. Behav. Sci. 2013, 75, 473-478. [CrossRef]

13. Kähkönen, K.; Keinänena, M.; Naaranoja, M. Core project teams as an organizational approach for projects and their management. Procedia Soc. Behav. Sci. 2012, 74, 369-376. [CrossRef]

14. Lander, M.W.; Koene, B.A.S.; Linssen, S.N. Committed to professionalism: Organizational responses of mid-tier accounting firms to conflicting institutional logics. Account. Organ. Soc. 2013, 38, 130-148. [CrossRef]

15. Douma, S.; Schreuder, H. Economic Approaches to Organizations, 5th ed.; Pearson Education Limited: Harlow, Essex, UK, 2013.

16. Mintzberg, H. Structure in Fives: Designing Effective Organizations; Prentice Hall: Upper Saddle River, NJ, USA, 1992.

17. Mintzberg, H. Tracking Strategies: Toward a General Theory of Strategy Formation; Oxford University Press: New York, NY, USA, 2009.

18. Lunenburg, F.C. Organizational structure: Mintzberg's framework. Int. J. Sch. Acad. Intellect. Divers. 2012, 14, 1-8.

19. Wikimedia Commons. Available online: https://commons.wikimedia.org/wiki/File:Technostructure.svg\#mw-head (accessed on 17 May 2021).

20. McCain, R.A. Game Theory: A Nontechnical Introduction to the Analysis of Strategy, revised ed.; World Scientific Publishing Co. Pte. Ltd.: Singapore, 2010.

21. Rubinstein, A.; Persson, K.G. Modeling Bounded Rationality; The MIT Press: Boston, MA, USA, 1998.

22. Kugler, T.; Kausel, E.E.; Kocher, M.G. Are groups more rational than individuals? A review of interactive decision making in groups. Wiley Interdiscip. Rev. Cogn. Sci. 2012, 3, 471-482. [CrossRef]

23. Markmann, C.; Darkow, I.; von der Gracht, H. A Delphi-based risk analysis-Identifying and assessing future challenges for supply chain security in a multi-stakeholder environment. Technol. Forecast. Soc. Chang. 2013, 80, 1815-1833. [CrossRef] 
24. Jianlei, Z.; Chunyan, Z.; Tianguang, C. Cooperation enhanced by the 'survival of the fittest' rule in prisoners' dilemma games on complex networks. J. Theor. Biol. 2010, 267, 41-47.

25. Jianlei, Z.; Xiaojie, C.; Chunyan, Z.; Long, W.; Tianguang, C. Elimination mechanism promotes cooperation in coevolutionary prisoners' dilemma games. Phys. A 2010, 389, 4081-4086.

26. Barron, E.N. Game Theory: An Introduction; Wiley-Interscience, John Wiley \& Sons Inc.: Hoboken, NJ, USA, 2011.

27. Zheng, T.; Wang, W.; Dong, W.; Yan, B.; Gou, J. Research on the Enterprise Safety and Low-Carbon Behaviors Management and Application Based on the Evolutionary Game. In Advances in Intelligent Systems and Computing, Proceedings of the Tenth International Conference on Management Science and Engineering Management, Singapore, 2017; Xu, J., Hajiyev, A., Nickel, S., Gen, M., Eds.; Springer: Berlin/Heidelberg, Germany, 2017.

28. Liu, J.; Gao, R.; Cheah, C.W.J.; Luo, J. Evolutionary game of investors' opportunistic behaviour during the operational period in PPP projects. Constr. Manag. Econ. 2017, 35, 137-153. [CrossRef]

29. Fei, D.; Yun, L.; Bo, S.; Xia-Meng, S. An evolutionary game theory model of binary opinion formation. Phys. A 2010, 389, 1745-1752.

30. Opricović, S. Compromise in cooperative game and the VIKOR method. Yugosl. J. Oper. Res. 2009, 19, 225-238. [CrossRef]

31. Reay, T.; Jones, C. Qualitatively capturing institutional logics. Strat. Org. 2016, 14, 441-454. [CrossRef]

32. Lukes, S. Power: A Radical View, 2nd ed.; Palgrave Macmillan: Hampshire, UK, 2005.

33. Coutinho, L.R.; Sichman, J.S.; Boissier, O. Modelling dimensions for agent organizations. In Handbook of Research on Multi-Agent Systems: Semantics and Dynamics of Organizational Models, 1st ed.; Dignum, V., Ed.; IGI Global: Hershey, PA, USA, 2009 ; pp. 18-50.

34. Arbnor, I.; Bjerke, B. Methodology for Creating Business Knowledge, 3rd ed.; Sage: Thousand Oaks, CA, USA, 2009.

35. Likert, R.; Bowers, D.G. Organizational theory and human resource accounting. Am. Psychol. 1969, 24, 585-592. [CrossRef]

36. Shafritz, J.M.; Ott, J.S.; Jang, Y.S. Classics of Organization Theory, 8th ed.; Cengage Learning: Boston, MA, USA, 2015.

37. Balsmeier, B.; Bermig, A.; Dilger, A. Corporate governance and employee power in the boardroom: An applied game theoretic analysis. J. Econ. Behav. Organ. 2013, 91, 51-74. [CrossRef]

38. Singh, S.; Tabassum, N.; Darwish, T.K.; Batsakis, G. Corporate Governance and Tobin's Q as a Measure of Organisational Performance. Br. J. Manag. 2018, 29, 171-190. [CrossRef]

39. Organ, D.W. Organizational Citizenship Behavior: The Good Soldier Syndrome; Lexington Books: Lexington, MA, USA, 1988.

40. Ebrahimpour, H.; Zahed, A.; Khaleghkhah, A.; Sepehri, M.B. A survey relation between organizational culture and organizational citizenship behavior. Procedia Soc. Behav. Sci. 2011, 30, 1920-1925. [CrossRef]

41. Wenzel, M.; Stanske, S.; Lieberman, M.B. Strategic responses to crisis. Strat. Manag. J. 2020, 41, V7-V18. [CrossRef]

42. Koch, C.; Sage, D.; Dainty, A.; Simonsen, R. Understanding operations strategizing in project-based organisations: Middle managers' interaction and strategy praxis. Eng. Proj. Org. J. 2015, 5, 106-117. [CrossRef]

43. Rezgui, Y.; Hopfe, C.J.; Vorakulpipat, C. Generations of knowledge management in the architecture, engineering and construction industry: An evolutionary perspective. Adv. Eng. Inform. 2010, 24, 219-228. [CrossRef]

44. Kulas, J.T.; Komai, M.; Grossman, P.J. Leadership, information and risk attitude: A game theoretic approach. Lead. Q 2013, 24, 349-352. [CrossRef]

45. Kefan, X.; Gang, C.; Wu, D.D.; Luo, C.; Qian, W. Entrepreneurial team's risk-based decision-making: A dynamic game analysis. Int. J. Prod. Econ. 2011, 134, 78-86. [CrossRef]

46. Shurong, Z.; Miao, P. Rent-seeking behaviors analysis in engineering supervision based on the game theory. Procedia Syst. Eng. 2012, 4, 455-459. [CrossRef]

47. Aquilani, B.; Silvestri, C.; Ruggieri, A.; Gatti, C. A systematic literature review on total quality management critical success factors and the identification of new avenues of research. TQM J. 2017, 29, 184-213. [CrossRef]

48. Standing, C.; Kiniti, S. How can organizations use wikis for innovation? Technovation 2011, 31, 287-295. [CrossRef]

49. Hwang, S. Sharing tacit knowledge in small-medium regional construction companies in the U.S.: The current status and the impact of organizational ecology. Int. J. Constr. Manag. 2020. [CrossRef]

50. Rwelamila, P.D.; Abdul-Aziz, A.R. Improving the Performance of Construction Industries for Developing Countries: Programmes, Initiatives, Achievements and Challenges, 1st ed.; Routledge: Abingdon, UK, 2021.

51. Sharma, R.S.; Bhattacharya, S. Knowledge dilemmas within organizations: Resolutions from game theory. Knowl. Based Syst. 2013, 45, 100-113. [CrossRef]

52. Konno, T. A condition for cooperation in a game on complex networks. J. Theor. Biol. 2011, 269, 224-233. [CrossRef]

53. Cressman, R.; Song, J.; Zhang, B.; Tao, Y. Cooperation and evolutionary dynamics in the public goods game with institutional incentives. J. Theor. Biol. 2012, 299, 144-151. [CrossRef]

54. Löwstedt, M.; Sandberg, R. Standardizing the free and independent professional: The case of construction site managers in Sweden. Eng. Constr. Arch. Manag. 2020, 27, 1337-1355. [CrossRef]

55. Reed, W.J. The Pareto, Zipf and other power laws. Econ. Lett. 2001, 74, 15-19. [CrossRef]

56. United Nations Development Program. 1992 Human Development Report; Oxford University Press: New York, NY, USA, 1992.

57. Wilkinson, L. Revising the Pareto chart. Am. Stat. 2006, 60, 332-334. [CrossRef]

58. Kämpke, T.; Radermacher, F.J. Pareto distribution, self-similarity and empirics. In Income Modeling and Balancing: A Rigorous Treatment of Distribution Patterns; Kämpke, T., Radermacher, F.J., Eds.; Springer International Publishing: Cham, Switzerland, 2014.

59. Bookstein, A. Informetric distributions, part I: Unified overview. J. Am. Soc. Inf. Sci. 1990, 41, 368-375. [CrossRef] 
60. Taleb, N.N. The Black Swan; Random House: New York, NY, USA, 2007.

61. Gastwirth, J.L. The estimation of the Lorenz Curve and Gini Index. Rev. Econ. Stat. 1972, 54, 306-316. [CrossRef]

62. European Commission. European Construction Sector Observatory: Country Profile Sweden; European Commission: Brussels, Belgium, 2020. 